\title{
ON THE INTERACTION BETWEEN SELF-EXCITED AND FORCED OSCILLATIONS
}

\author{
Thai Manh Cau, NguYen Van Khang \\ Hanoi University of Technology
}

\begin{abstract}
The influence of the exciting force to resonant characteristics in a model of the small oscillation is investigated. Averaging method is used to determine resonant stationary solutions of all possible resonant oscillations and their stability. The numerical simulations used for oscillations in time domain and the analytic approaches give consistent results.
\end{abstract}

\section{Introduction}

Investigation of non-linear problems in dynamics of machines is actual in engineering practice. Interaction of various kinds of non-linear oscillations is one of significant technical problems. Some researches are shown in $[1,2,3]$. The differential equation of some oscillatory models in machines is written in the form

$$
\ddot{x}-b_{1} \dot{x}+b_{2} \dot{x}^{2}+b_{3} \dot{x}^{3}+\gamma_{2} x^{2}+\gamma_{3} x^{3}+\omega_{0}^{2} x-B_{0}=E \cos \Omega t .
$$

This paper presents an examination on the influence of the exciting force to resonant characteristics in a model of the small oscillation. Averaging method and numerical simulation are used to determine stationary solutions and their stability. Numerical simulations by using the MATLAB program are applied.

2. Averaging Method

Assume the oscillation to be small, let $x_{0}$ be a root of the equation

$$
\gamma_{3} x^{3}+\gamma_{2} x^{2}+\omega_{0}^{2} x-B_{0}=0 .
$$

By using the new variable

$$
u=x-x_{0}
$$

the equation (1.1) is transformed into the following form

$$
\ddot{u}+\omega^{2} u=\varepsilon f(u, \dot{u})+E \cos \Omega t,
$$

where $\omega^{2}=3 \gamma_{3} x_{0}^{2}+2 \gamma_{2} x_{0}+\omega_{0}^{2}$

$\varepsilon$ is a formal parameter indicating the smallness of the right side, 
$\varepsilon f(u, \dot{u})=-\gamma_{2}^{*} u^{2}-\gamma_{3} u^{3}+b_{1} \dot{u}-b_{2} \dot{u}^{2}-b_{3} \dot{u}^{3}$,

and $\gamma_{2}^{*}=\gamma_{2}+3 \gamma_{3} x_{0}$.

\subsection{Case of small exciting force}

Assume the exciting force to be small. Let transform the time $t$ into a nondimensional quantity $\tau$,

$$
\tau=\frac{\Omega}{m} t
$$

where $m$ is some positive integer.

The equation (2.1) becomes

$$
u^{\prime \prime}+\lambda u=\varepsilon\left[f\left(u, u^{\prime}\right)+E_{0} \cos m \tau\right],
$$

where

$$
\begin{aligned}
& \lambda=\frac{m^{2}}{\Omega^{2}} \omega^{2}, \quad g_{2}=\frac{m^{2}}{\Omega^{2}} \gamma_{2}^{*}, \quad g_{3}=\frac{m^{2}}{\Omega^{2}} \gamma_{3}, \\
& \beta_{1}=\frac{m}{\Omega} b_{1}, \quad \beta_{2}=b_{2}, \quad \beta_{3}=\frac{\Omega}{m} b_{3}, \quad E_{0}=\frac{m^{2}}{\Omega^{2}} E, \\
& f\left(u, u^{\prime}\right)=-g_{2} u^{2}-g_{3} u^{3}+\beta_{1} u^{\prime}-\beta_{2} u^{\prime 2}-\beta_{3} u^{\prime 3} .
\end{aligned}
$$

In order to examine resonant regimes of the system, one assumes

$$
\lambda=n^{2}(1-\varepsilon \alpha),
$$

where $n$ is some positive parameter, $\alpha$ is a detuning parameter.

The equation (2.3) has the form

$$
u^{\prime \prime}+n^{2} u=\varepsilon\left[n^{2} u \alpha+f\left(u, u^{\prime}\right)+E_{0} \cos m \tau\right] .
$$

A solution of (2.5) is found in the form

$$
u=r \cos n \tau+s \sin n \tau, \quad u^{\prime}=-n r \sin n \tau+n s \cos n \tau,
$$

where $r(\tau)$ and $s(\tau)$ are unknown functions of $\tau$.

Averaging equations have the form

$$
\begin{aligned}
& \frac{d r}{d t}=-\frac{\varepsilon}{2 n}\left\{n^{2} s \alpha-\frac{3}{4} g_{3}\left(r^{2}+s^{2}\right) s-n \beta_{1} r+\frac{3}{4} \beta_{3} n^{3}\left(r^{2}+s^{2}\right) r\right\}, \\
& \frac{d s}{d t}=\frac{\varepsilon}{2 n}\left\{n^{2} r \alpha-\frac{3}{4} g_{3}\left(r^{2}+s^{2}\right) r+n \beta_{1} s-\frac{3}{4} n^{3} \beta_{3}\left(r^{2}+s^{2}\right) s+E_{0} \delta_{m}^{n}\right\},
\end{aligned}
$$

where $\delta_{m}^{n}$ is Kronecker symbol. 
It is worth mentioning that there is only resonant case when $m=n$ in this case. It is main resonance $\omega \approx \Omega$.

The stationary oscillation $\left(r=r_{0}=\right.$ const, $s=s_{0}=$ const $)$ satisfies the system of equations

$$
\begin{aligned}
& \Phi_{1}(r, s)=0, \\
& \Phi_{2}(r, s)=0,
\end{aligned}
$$

where

$$
\begin{aligned}
& \Phi_{1}(r, s)=-\frac{1}{2 n}\left\{n^{2} s \alpha-\frac{3}{4} g_{3}\left(r^{2}+s^{2}\right) s-n \beta_{1} r+\frac{3}{4} \beta_{3} n^{3}\left(r^{2}+s^{2}\right) r\right\}, \\
& \Phi_{2}(r, s)=\frac{1}{2 n}\left\{n^{2} r \alpha-\frac{3}{4} g_{3}\left(r^{2}+s^{2}\right) r+n \beta_{1} s-\frac{3}{4} \beta_{3} n^{3}\left(r^{2}+s^{2}\right) s+E_{0}\right\},
\end{aligned}
$$

Denote $A^{2}=r^{2}+s^{2}$, from (2.8) one gets the amplitude-frequency formula of resonant curve $(\Gamma)$

$$
n^{2} \alpha=\frac{3}{4} g_{3} A^{2} \pm \sqrt{\frac{E_{0}^{2}}{A^{2}}-\left(\beta_{1} n-\frac{3}{4} \beta_{3} n^{3} A^{3}\right)^{2}} .
$$

Let $\left(\alpha^{+}\right)$and $\left(\alpha^{-}\right)$be branches of $(\Gamma)$ on the plane $(\alpha, A)$ when the sign, which is before the symbol of square root, is "+" or " - ", respectively.

Conditions of asymptotic stability of stationary oscillation are

$$
\begin{aligned}
& a_{11}+a_{22}<0, \\
& a_{11} a_{22}-a_{12} a_{21}>0 .
\end{aligned}
$$

where

$$
a_{11}=\left.\frac{\partial \Phi_{1}}{\partial r}\right|_{\left(r_{0}, s_{0}\right)}, \quad a_{12}=\left.\frac{\partial \Phi_{1}}{\partial s}\right|_{\left(r_{0}, s_{0}\right)}, \quad a_{21}=\left.\frac{\partial \Phi_{2}}{\partial r}\right|_{\left(r_{0}, s_{0}\right)}, \quad a_{22}=\left.\frac{\partial \Phi_{2}}{\partial s}\right|_{\left(r_{0}, s_{0}\right)} .
$$

By calculating from (2.8), (2.9) one obtains

$$
\begin{aligned}
a_{11}+a_{22} & =\beta_{1}-\frac{3}{2} \beta_{3} n^{3} A^{2}, \\
a_{11} a_{22}-a_{12} a_{21} & =\frac{1}{4 n^{2}}\left\{\frac{E_{0}^{2}}{A^{2}}-\frac{3}{2} \beta_{3} n^{2} A^{2}\left(n \beta_{1}-\frac{3}{4} \beta_{3} n^{3} A^{2}\right)-\frac{3}{2} g_{3} A^{2}\left(n^{2} \alpha-\frac{3}{4 g_{3} A^{2}}\right)\right\} \\
& =-\frac{A^{2}}{4 n^{4}}\left\{ \pm \sqrt{\frac{E_{0}^{2}}{A^{2}}-\left(n \beta_{1}-\frac{3}{4} \beta_{3} n^{3} A^{2}\right)^{2}}\right\} \frac{d \alpha}{d A} .
\end{aligned}
$$

Hence, the condition (2.11) depends on $\frac{d \alpha}{d A}$. Geometrically, on the plane $(\alpha, A)$ stationary oscillations correspond to the points of the resonant curve. For the branch 
$\left(\alpha^{+}\right)$the condition of stability is $\frac{d \alpha}{d A}<0$, i. e. the part sloping down corresponds to stable stationary oscillations. For the branch $\left(\alpha^{-}\right)$, the condition of stability is $\frac{d \alpha}{d A}>0$, i. e. the part sloping up corresponds to stable stationary oscillations.

Denote by $(C)$ a line $a_{11}+a_{22}=0$. The condition (2.10) satisfies for the part of ( $\Gamma)$ lying upon or below $(C)$ if $\beta_{3}>0$ or if $\beta_{3}<0$, respectively.

Fig. 1 shows the resonant curve for parameters: $g_{2}=0.01, g_{3}=0.001, b_{1}=0.5$, $b_{2}=0.01, b_{3}=0.1, E=0.5, \omega=40, \Omega=40$. The solid part of the curve corresponds to stable regimes, the dot one corresponds to unstable regimes.

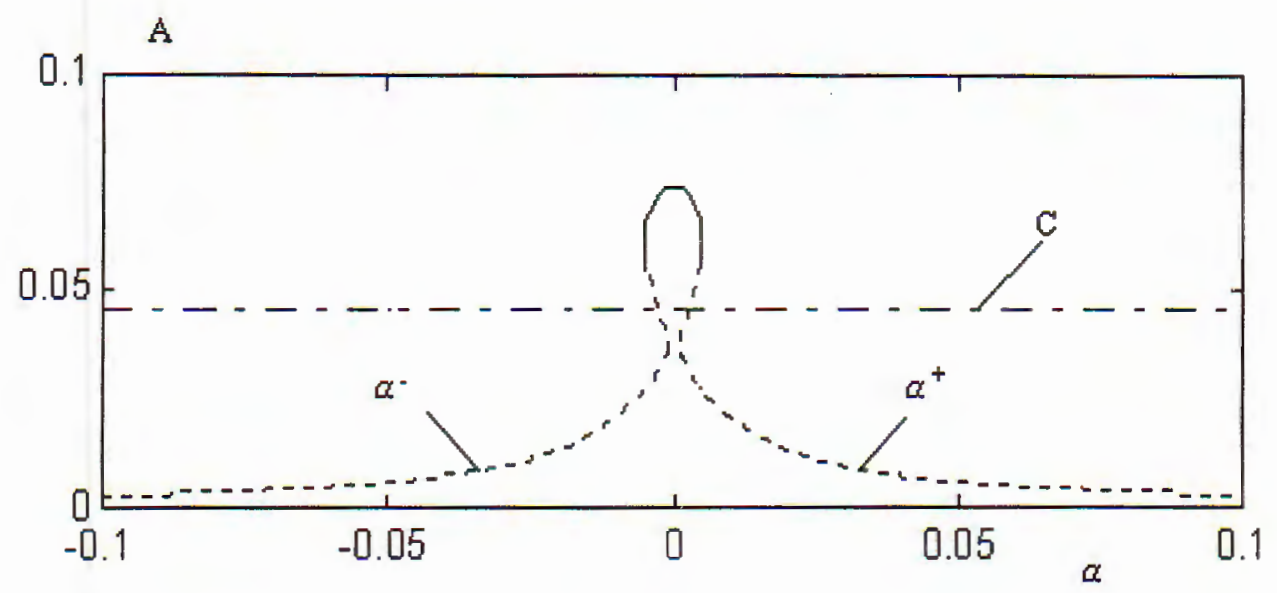

Fig. $1(\omega=\Omega)$

\subsection{Case of finite exciting force}

Suppose that exciting force is finite, i. e. $E_{0}$ is not a small quantity. The differential equation of oscillation has the form

$$
u^{\prime \prime}+\lambda u=\varepsilon f\left(u, u^{\prime}\right)+E_{0} \cos m \tau,
$$

where $\lambda$ and $f\left(u, u^{\prime}\right)$ are determined by (2.4).

In order to examining resonant regimes of the system, one assumes

$$
\lambda=n^{2}(1-\varepsilon \alpha),
$$

where $n$ is some positive integer, $\alpha$ is a detuning parameter.

The equation (2.15) becomes

$$
u^{\prime \prime}+n^{2} u=\varepsilon\left[n^{2} \alpha u+f\left(u, u^{\prime}\right)\right]+E_{0} \cos m \tau .
$$

A solution of (2.16) is found in the form

$$
\begin{aligned}
u & =r \cos n \tau+s \sin n \tau+e \cos m \tau, \\
u^{\prime} & =-n r \sin n \tau+n s \cos n \tau-m e \sin m \tau,
\end{aligned}
$$


where $e=\frac{E_{0}}{n^{2}-m^{2}},(n \neq m)$ and $r=r(\tau), s=s(\tau)$ are unknown functions of $\tau$.

Averaging equations have the form

$$
\begin{aligned}
& \frac{d r}{d t}=\varepsilon \Phi_{1}(r, s), \\
& \frac{d s}{d \tau}=\varepsilon \Phi_{2}(r, s),
\end{aligned}
$$

where

$$
\begin{aligned}
& \Phi_{1}(r, s)^{6}=-\frac{1}{2 n}\left\{n^{2} \alpha s+g_{2} e s \delta_{m}^{2 n}-\frac{3}{4} g_{3}\left[s\left(r^{2}+s^{2}\right)-2 e r s \delta_{m}^{3 n}+2 e^{2} s\right]-\beta_{1} n r\right. \\
&\left.+\beta_{2} n m e s \delta_{m}^{2 n}+\frac{3}{4} \beta_{3}\left[n^{3} r\left(r^{2}+s^{2}\right)-\frac{1}{3} e^{3} m^{3} \delta_{3 m}^{n}+2 n m^{2} e^{2} r+n^{2} m e\left(s^{2}-r^{2}\right) \delta_{m}^{3 n}\right]\right\} \\
& \Phi_{2}(r, s)= \frac{1}{2 n}\left\{n^{2} \alpha r-\frac{1}{2} g_{2}\left(e^{2} \delta_{2 m}^{n}+2 e r \delta_{m}^{2 n}\right)-\frac{3}{4} g_{3}\left[r\left(r^{2}+s^{2}\right)\right.\right. \\
&\left.+\frac{1}{3} e^{3} \delta_{3 m}^{n}-e s^{2} \delta_{m}^{3 n}+e r^{2} \delta_{m}^{3 n}+2 e^{2} r\right]+\beta_{1} n s+\beta_{2}\left[m^{2} e^{2} \delta_{2 m}^{n}-n m e r \delta_{m}^{2 n}\right] \\
&\left.-\frac{3}{4} \beta_{3}\left[n^{3} s\left(r^{2}+s^{2}\right)+2 n^{2} \operatorname{mers} \delta_{m}^{2 n}+2 n m^{2} e^{2} s\right]\right\}
\end{aligned}
$$

The stationary oscillation ( $r=r_{0}=$ const, $s=s_{0}=$ const) is the solution of the equation

$$
\begin{aligned}
& {\left[n^{2} \alpha-\frac{3}{4} g_{3}\left(A^{2}+2 e^{2}\right)\right]\left[\begin{array}{l}
s \\
r
\end{array}\right]-\left[\frac{3}{4} \beta_{3} n\left(n^{2} A^{2}+2 e^{2} m^{2}\right)-\beta_{1} n\right]\left[\begin{array}{c}
-r \\
s
\end{array}\right]+} \\
& +\left(g_{2} e+\beta_{2} n m e\right)\left[\begin{array}{c}
s \\
-r
\end{array}\right] \delta_{m}^{2 n}-\left(\frac{1}{2} g_{2} e^{2}-m^{2} e^{2}\right)\left[\begin{array}{l}
0 \\
1
\end{array}\right] \delta_{2 m}^{n}-\frac{e^{3}}{4}\left[\begin{array}{c}
\beta_{3} m^{3} \\
g_{3}
\end{array}\right] \delta_{3 m}^{n} \\
& +\frac{3}{4} g_{3} e\left[\begin{array}{c}
2 r s \\
s^{2}-r^{2}
\end{array}\right] \delta_{m}^{3 n}+\frac{3}{4} \beta_{3} n^{2} m e\left[\begin{array}{c}
s^{2}-r^{2} \\
-2 r s
\end{array}\right] \delta_{m}^{3 n}=0
\end{aligned}
$$

where $\delta_{i}^{j}$ is Kronecker symbol and $A=\sqrt{r^{2}+s^{2}}$.

It is worth mentioning that there are resonant cases of the system when $m=2 n$, $m=n / 2, m=3 n, m=n / 3$.

For $A \neq 0$ it follows from (2.20) a formula relating amplitude to frequency. The results obtained are recapitulated in the Table 1.

Now we determine the stability conditions of the resonant oscillations. By using conditions of asymptotic stability (2.10) and (2.11) one can establish the stable conditions for stationary solutions.

a) Case of $m=2 n$. For stationary solution $A \neq 0$ the stability conditions are

$$
\beta_{1}-\frac{3}{2} \beta_{3} n^{2}\left(A^{2}+4 e^{2}\right)<0, \quad \frac{d \alpha}{d A}<0 \quad \text { or } \quad \frac{d \alpha}{d A}>0
$$


for the sign before the symbol of square root in amplitude-frequency formula is positive or negative, respectively.

For the trivial solution $A=0$, the stability condition is

$$
\beta_{1}-6 \beta_{3} n^{2} e^{2}<0
$$

b) Case of $m=n / 2$. For stationary solution $A \neq 0$ the stability conditions are

$$
\beta_{1}-\frac{3}{2} \beta_{3} n^{2}\left(A^{2}+\frac{e^{2}}{4}\right)<0, \quad \frac{d \alpha}{d A}<0 \quad \text { or } \quad \frac{d \alpha}{d A}>0,
$$

for the sign before the symbol of square root in the amplitude-frequency formula is positive or negative, respectively.

The trivial solution $A=0$ does not exist.

c) Case of $m=3 n$. For stationary solution $A \neq 0$ the stability conditions are

$$
\beta_{1}-\frac{3}{4} \beta_{3} n^{2}\left(2 A^{2}+9 e^{2}\right)<0, \quad \frac{d \alpha}{d A}<0 \quad \text { or } \quad \frac{d \alpha}{d A}>0,
$$

for the sign before the symbol of square root in the amplitude-frequency formula is positive or negative, respectively.

For the trivial solution $A=0$, the stability condition is

$$
\beta_{1}-\frac{27}{4} \beta_{3} n^{2} e^{2}<0 \text {. }
$$

d) Case of $m=n / 3$. For stationary solution $A \neq 0$ the stability conditions are

$$
\beta_{1}-\frac{3}{2} \beta_{3} n^{2}\left(A^{2}+\frac{e^{2}}{9}\right)<0, \quad \frac{d \alpha}{d A}<0 \quad \text { or } \quad \frac{d \alpha}{d A}>0,
$$

The trivial solution $A=0$ does not exist.

Table 1

\begin{tabular}{lc}
\hline $\begin{array}{c}\text { Resonance } \\
\text { possi- } \\
\text { bilities }\end{array}$ & Amplitude-frequency formulas \\
$\frac{m=2 n}{m=2 n}$ & $n^{2} \alpha=\frac{3}{4} g_{3}\left(A^{2}+2 e^{2}\right) \pm \sqrt{\left(g_{2}+2 \beta_{2} n^{2}\right) e^{2}-\left[\beta_{1} n-\frac{3}{4} \beta_{3} n^{3}\left(A^{2}+8 e^{2}\right)\right]^{2}}$ \\
$\left(\omega \approx \frac{\Omega}{2}\right)$ & \\
$m=n / 2$ & $n^{2} \alpha=\frac{3}{4} g_{1}\left(A^{2}+2 e^{2}\right) \pm \sqrt{\left(g_{2}-\frac{1}{2} \beta_{2} n^{2}\right) \frac{e^{4}}{4 A^{2}}-\left[\beta_{1} n-\frac{3}{4} \beta_{3} n^{3}\left(A^{2}+\frac{e^{2}}{2}\right)\right]^{2}}$ \\
$(\omega \approx 2 \Omega)$ & \\
$m=3 n$ & $n^{2} \alpha=\frac{3}{4} g_{3}\left(A^{2}+2 e^{2}\right) \pm \sqrt{\left(\frac{3 e A}{4}\right)^{2}\left(g_{3}^{2}+9 \beta_{3}^{2} n^{6}\right)-\left[\beta_{1} n-\frac{3}{4} \beta_{3} n^{3}\left(A^{2}+9 e^{2}\right)\right]^{2}}$ \\
$\left(\omega \approx \frac{\Omega}{3}\right)$ & \\
$m=n / 3$ & $n^{2} \alpha=\frac{3}{4} g_{3}\left(A^{2}+2 e^{2}\right) \pm \sqrt{\left(\frac{e^{3}}{4 A}\right)^{2}\left(g_{3}^{2}+\frac{1}{27^{2}} \beta_{3}^{2} n^{6}\right)-\left[\beta_{1} n-\frac{3}{4} \beta_{3} n^{3}\left(A^{2}+\frac{2}{9} e^{2}\right)\right]^{2}}$ \\
$(\omega \approx 3 \Omega)$ &
\end{tabular}


Fig. 1 shows the resonant curve for parameters: $g_{2}=0.01, g_{3}=0.001, b_{1}=0.5$, $b_{2}=0.01, b_{3}=0.1, E=0.5, \omega=40, \Omega=40$. The resonant curve for parameters: $g_{2}=0.04, g_{3}=0.001, b_{1}=0.03, b_{2}=0.001, b_{3}=0.005, E=10, \omega=4, \Omega=2$ is shown on Fig. 2. Fig. 3 shows the resonant curve for parameters: $g_{2}=0.01$, $g_{3}=0.001, b_{1}=0.02, b_{2}=0.0001, b_{3}=0.03, E=5, \omega=6, \Omega=2$. On Fig. 4 the resonant curve for parameters: $g_{2}=0.3, g_{3}=0.3, b_{1}=0.003, b_{2}=0.0001$, $b_{3}=0.0005, E=50, \omega=4, \Omega=8$ is shown. Fig. 5 shows the resonant curve for parameters: $g_{2}=0.01, g_{3}=0.3, b_{1}=0.001, b_{2}=0.0001, b_{3}=0.005, E=50, \omega=4$, $\Omega=12$. The solid parts of the curves correspond to stable regimes, the dot ones correspond to unstable regimes.

A

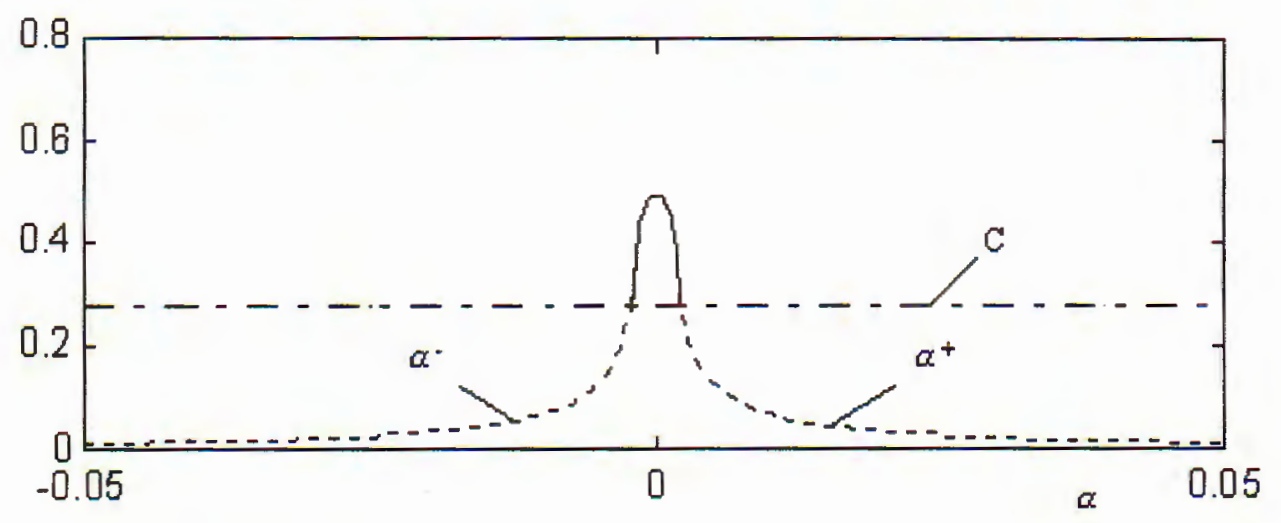

Fig. $2(\omega=\Omega / 2)$

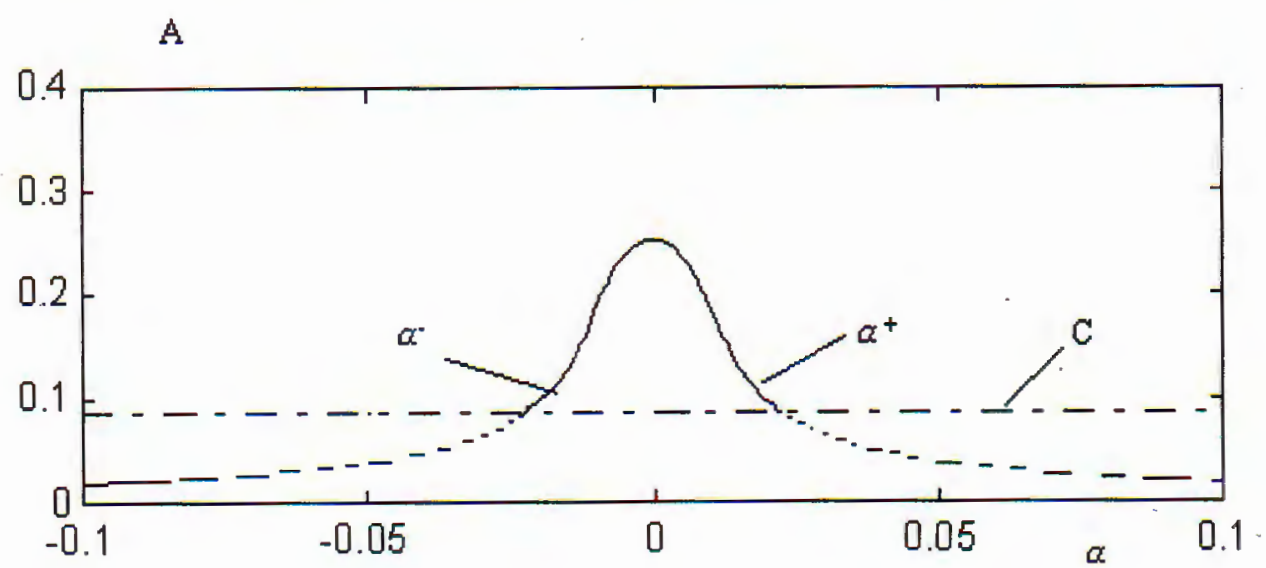

Fig. $3(\omega=\Omega / 3)$ 


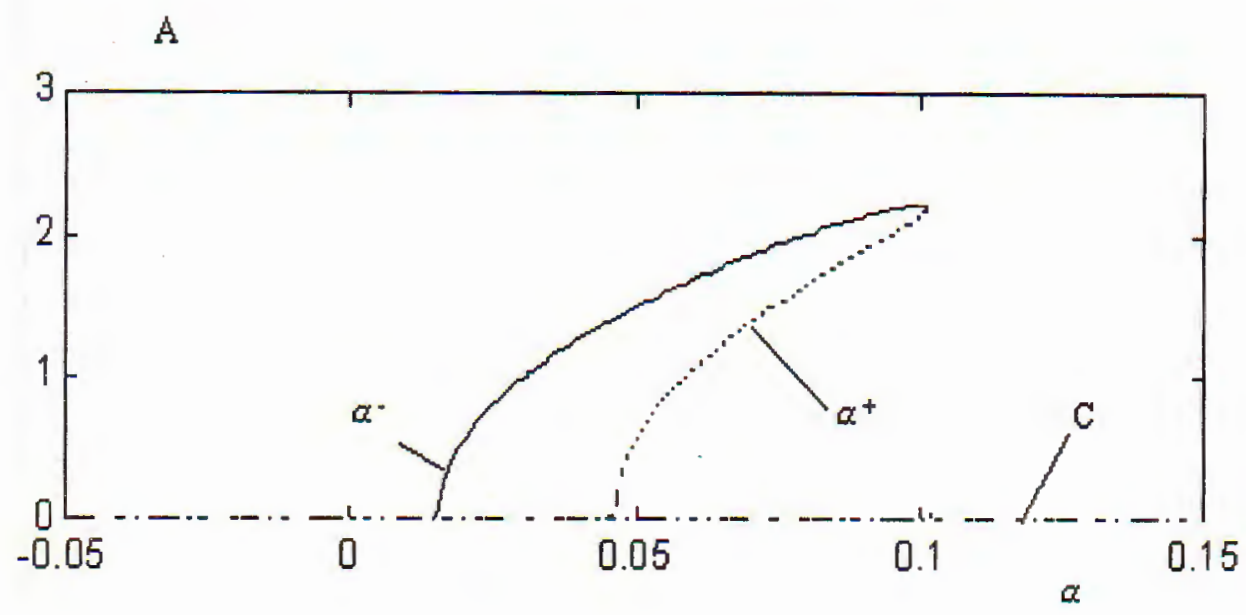

Fig. $4(\omega \approx 2 \Omega)$

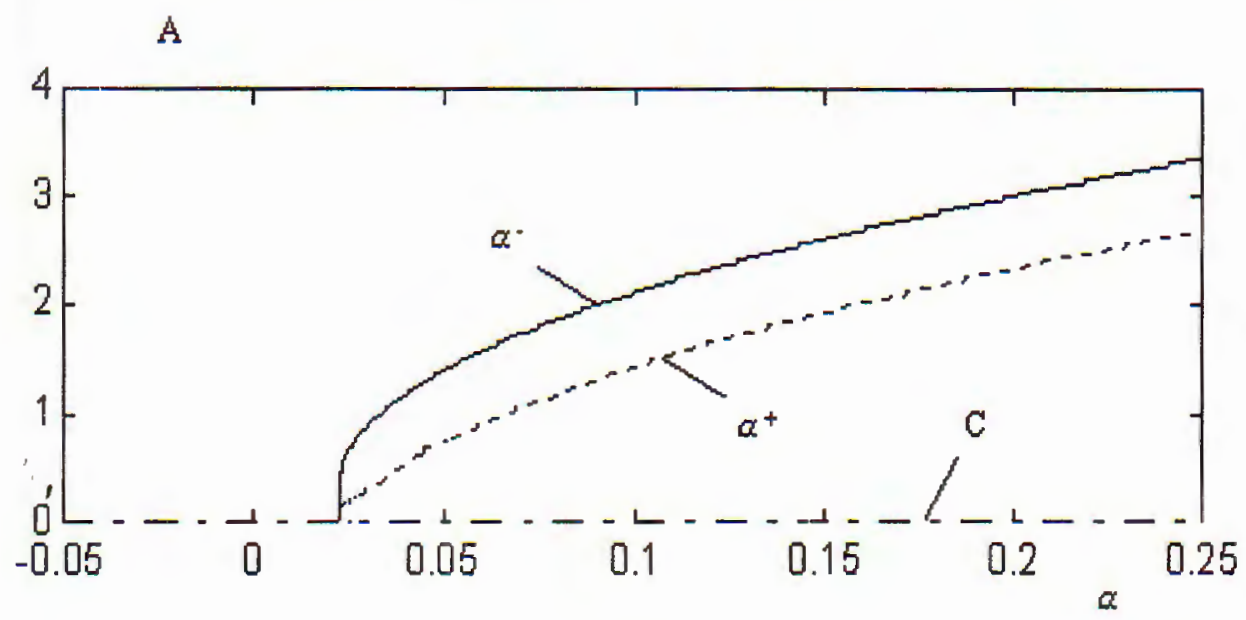

Fig. $5(\omega \approx 3 \Omega)$

\section{Numerical Simulations}

By using MATLAB program [10], we integrate the differential equation of oscillation (2.1) with the parameter determined from the amplitude-frequency curves, respectively.

Fig. $6,7,8,9,10$ represent the oscillations in time domain that correspond to parameters used in fig. $1,2,3,4,5$. The oscillations shown on fig. 6, 7, 8 correspond to $\alpha=0$, but ones on fig. 9, 10 correspond to $\alpha=0.05$. The figures a) show displacement-time and figures b): phase orbits, respectively. 


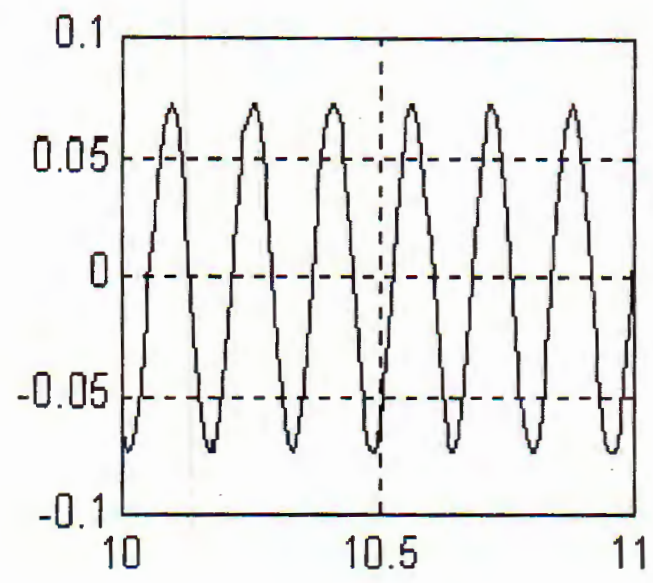

a)

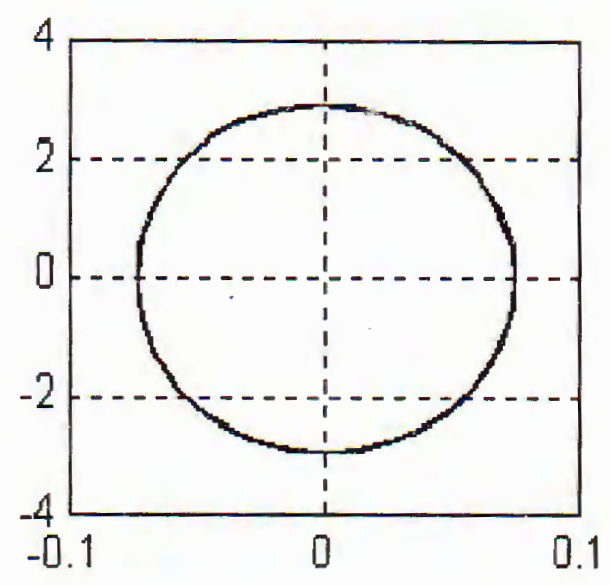

b)

Fig. $6(\omega=\Omega)$

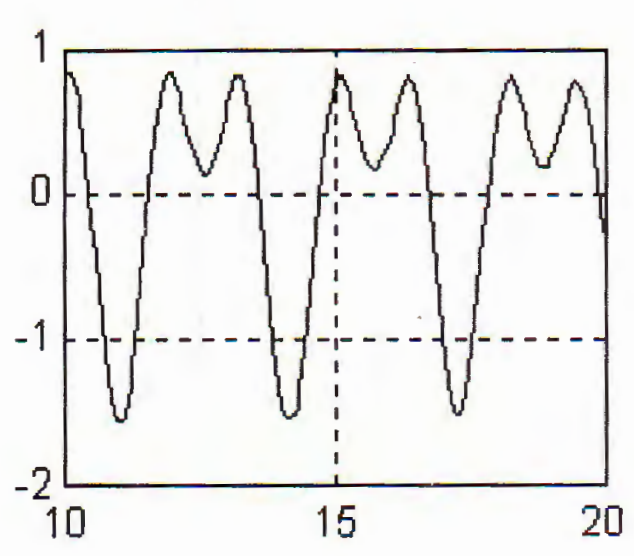

a)

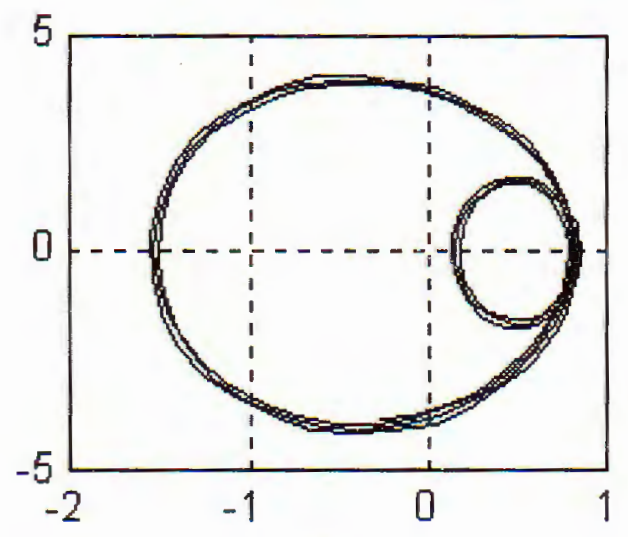

b)

Fig. $7(\omega=\Omega / 2)$

\section{Conclusion}

This paper presents an application of averaging method for examination of the influence of the exciting force to resonant characteristics in a model of the small oscillation. An analytic approach has provided amplitude-frequency equations and enabled to examine stability regimes of possible resonant oscillations in cases of small and finite exciting forces.

The numerical simulations used for oscillations in time domain and the analytic approaches give consistent results. Further investigations of influence of parameters on oscillation regimes of dynamics of machine will be published later.

This work is completed with financial support from the Council for Natural Science of Vietnam. 


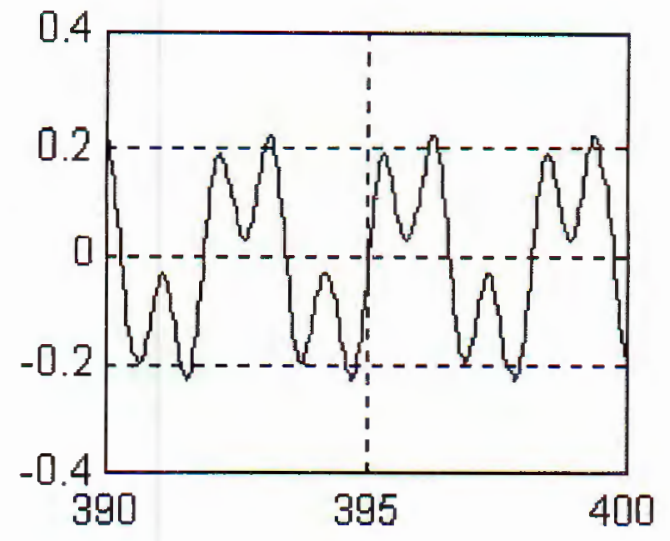

a)

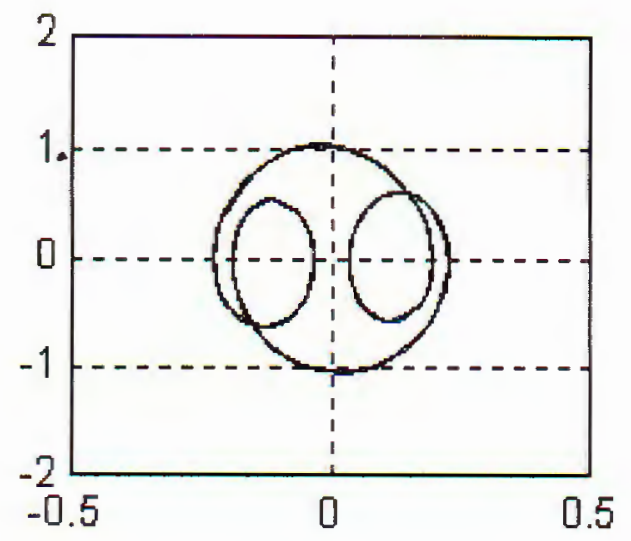

b)

Fig. $8(\omega=\Omega / 3)$

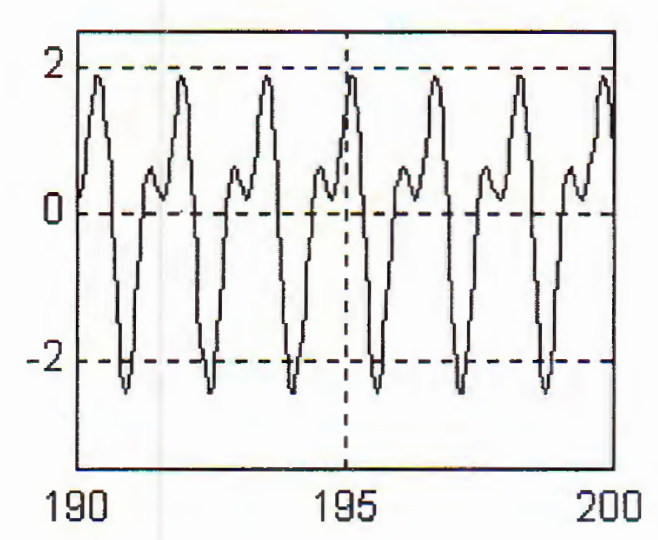

a)

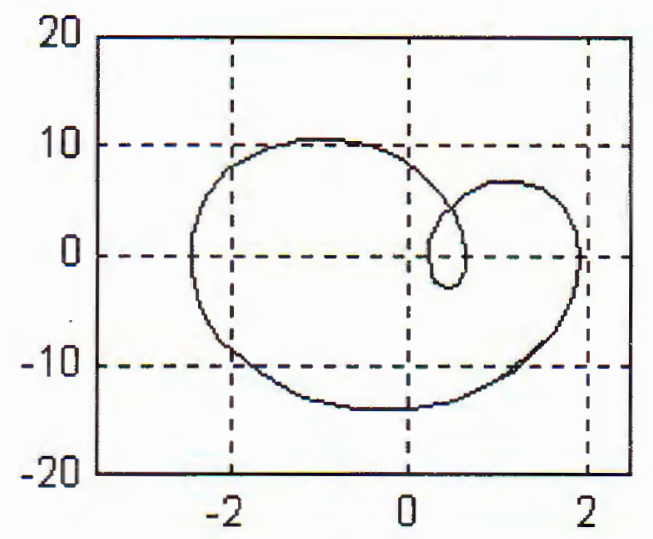

b)

Fig. $9(\omega \approx 2 \Omega)$

\section{REFERENCES}

1. Bogoliubov N. N., Mitropolskii Yu. A. Asymptotic Method in the Theory of Nonlinear Oscillations. 4th ed. (in Russian), Moscow 1974.

2. Mitropolskii Yu. A., Nguyen Van Dao. Applied Asymptotic Methods in Nonlinear Oscillations. Kluwer, Dordrecht 1997.

3. Schmidt G. Parameterregte Schwingungen. Deutscher Verlag der Wissenschaften, Berlin 1975.

4. Nguyen Van Khang, Tran Dinh Son. Influence of Non-linear Parametric Excitation on Resonant Characteristics of Oscillating Systems. Vietnam Journal of Mechanics, NCNST of Vietnam T. XX, 1998, No 2 (27-36). 


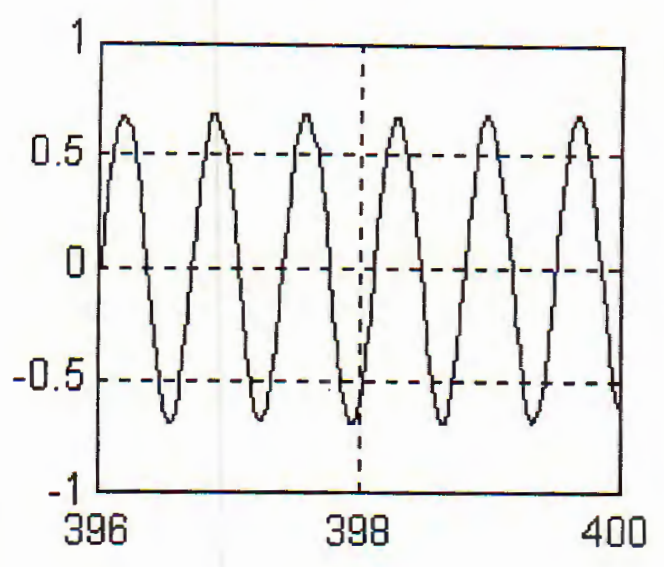

a)

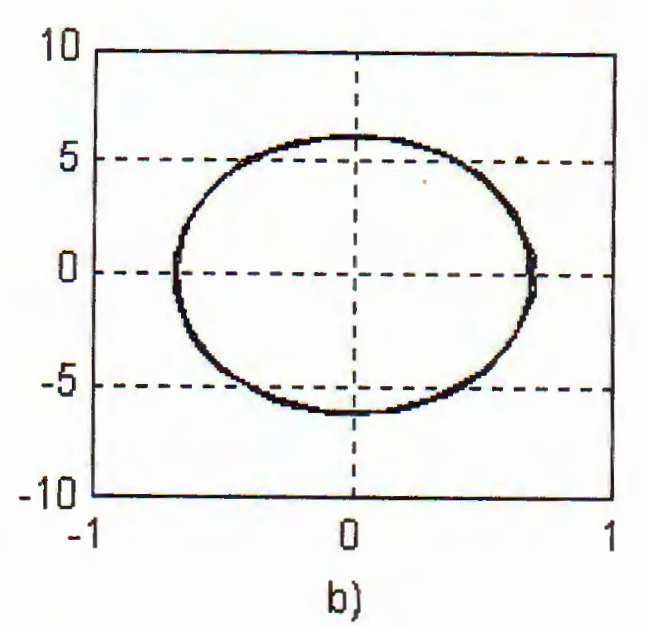

b)

Fig. $10(\omega \approx 3 \Omega)$

5. Tondl A. Nonlinear Oscillations of Mechanical Systems. Mir Publisher Moscow 1973.

6. Guckenheimer J., Holmes Ph. Nonlinear Oscillations, Dynamical Systems, and Bifurcations of Vector Fields. Springer, New York 1983.

7. Muraskin L. S., Muraskin S. L. Applied Nonlinear Oscillations of Tool Machines (in Russian), Saint Peterburg 1977.

8. Danek O., Polacek M., Spacek L., Tlusty J. Selbsterregte Schwingungen an Werkzeugmaschinen. Verlag Technik, Berlin 1962.

9. Bành Tiến Long, Trần Sỹ Túy, Trịnh Văn Tự. Nguyên lý gia công vật liệu. NXB Khoa học và Kỹ thuật, Hà Nội 2001.

10. Brian A., Breiner M. MATLAB für Ingenieure. Addison - Wesley'Publishing Company, Bonn 1995.

Received November 5, 2001

\section{VỀ TƯONG TÁC GIŨ̃A DAO ĐộNG TƯ KÍCH VÀ CỮNG BỨC}

Bài báo trình bày ảnh hưởng của lực kích động đến đặc trưng cộng hưởng của một mô hình dao động bé thường gặp trong dao động máy phi tuyến. Sứ dụng phương pháp trung bình để xác định phương trình của đường cong cộng hưởng và khảo sát các chế độ ổn định của tất cấ các dao động cộng hường có thể xầy ra. Các mô phơng số cho kết quả khá phù hợp với tính toán bằng giải tích. 